\title{
Modelagem espacial da hanseníase no estado da Bahia, Brasil, (2001-2015) e determinantes sociais da saúde
}

\author{
Spatial modeling of leprosy in the state of Bahia, Brazil, (2001-2015) \\ and social determinants of health
}

Carlos Dornels Freire de Souza (https://orcid.org/0000-0001-7995-1893) ${ }^{1}$

Roberto de Andrade Medronho (https://orcid.org/0000-0002-4617-6904) ${ }^{2}$

Franklin Gerônimo Bispo Santos (https://orcid.org/0000-0001-7007-4644) ${ }^{1}$

Mônica de Avelar Figueiredo Mafra Magalhães (https://orcid.org/0000-0002-6595-8274) ${ }^{3}$

Carlos Feitosa Luna (https://orcid.org/0000-0001-9277-4086) ${ }^{4}$

${ }^{1}$ Universidade Federal de Alagoas. Rodovia AL-115, Bom Sucesso. 57309-005 Arapiraca AL Brasil. carlos.freire@

arapiraca.ufal.br

${ }^{2}$ Faculdade de Medicina,

Universidade Federal do Rio

de Janeiro. Rio de Janeiro

RJ Brasil.

${ }^{3}$ Instituto de Comunicação

e Informação Científica

e Tecnológica em Saúde,

Fiocruz. Rio de Janeiro RJ

Brasil.

${ }^{4}$ Núcleo de Estatística

e Geoprocessamento,

Centro de Pesquisas Aggeu

Magalhães, Fiocruz. Recife

PE Brasil.

\begin{abstract}
This work analyzes the spatial distribution of leprosy in Bahia and associated social determinants. It is an ecological study, with leprosy data from 2001-2015. Three epidemiological indicators were selected: coefficient of detection in the general population and in children under 15 and the rate of new cases with grade II physical disability. These indicators were flattened by the Local Empirical Bayesian Model and Global and Local Moran statistics were applied. The independent variables were selected from the IBGE-2010 Census. Multivariate regressions were employed, followed by spatial regression. Leprosy exhibited a heterogeneous distribution in the state, with concentration in the north-west axis and the south region. For the general detection coefficient, five variables composed the final model: demographic density, urban population proportion, per capita income, proportion of extremely poor and households with over three people per dormitory. The illiteracy proportion made up the final model for the grade II rate of physical disability. No determinants of the occurrence of the disease were identified in children under 15. The modeling used contributed to demonstrate the spatial heterogeneity and social determinants of the disease in Bahia, revealing the complexity of the problem. Key words Leprosy, Social determinants of health, Spatial analysis.
\end{abstract}

Resumo O trabalho analisa a distribuição espacial da hanseníase na Bahia e os determinantes sociais relacionados. Estudo ecológico com dados de hanseníase do período 2001-2015. Três indicadores epidemiológicos foram selecionados: coeficiente de detecção na população geral e em menores de 15 anos e a taxa de casos novos com grau II de incapacidade. Os indicadores foram suavizados pelo Modelo Bayesiano Empírico Local e aplicou-se estatística de Moran Global e Local. As variáveis independentes foram selecionadas a partir do Censo IBGE-2010. Regressões multivariadas foram empregadas, seguidas de regressão espacial. Observou-se distribuição heterogênea no estado, com concentração no eixo norte-oeste e região sul. Para o coeficiente de deteç̧ão geral, cinco variáveis compuseram o modelo: densidade demográfica, proporção da população urbana, renda per capita, proporção de extremamente pobres e domicílios com mais de três pessoas por dormitório. A proporção de analfabetismo compôs o modelo final para a taxa de grau II de incapacidade física. Não foram identificados determinantes da ocorrência da doença em menores de 15 anos. A modelagem utilizada contribuiu para demonstrar a heterogeneidade espacial e os determinantes sociais da doença na Bahia, colocando em evidência a complexidade do problema.

Palavras-chave Hanseníase, Determinantes sociais da saúde, Análise espacial 


\section{Introdução}

A relação entre as condições sociais e econômicas e a saúde humana não é um tema recente na ciência. Desde séculos passados já se buscavam meios para compreender essa complexa relaçã $0^{1-3}$. A necessidade de entender de que modo os fatores econômicos, sociais, políticos, culturais e geográficos afetam a saúde humana, influenciando o desenvolvimento de determinadas doenças numa população, começou a ganhar espaço no mundo científico e político, que assumiu o processo saúde-doença como polissêmico, dinâmico e complexo, não se reduzindo a fatores biológi$\cos ^{1-4}$. Um grupo dessas doenças, chamadas de negligenciadas, como a hanseníase, a tuberculose, a leishmaniose e outras tantas, guarda estreita relação com as condições socioeconômicas e com o modo de ocupação do espaço geográfico ${ }^{3-5}$.

A hanseníase é uma doença infecciosa, crônica e granulomatosa causada pelo Mycobacterium leprae. Trata-se de um bacilo Álcool-Ácido Resistente (BAAR) transmitido de pessoa a pessoa por via respiratória e que tem grande afinidade pelas células cutâneas e nervosas periféricas, resultando em lesões de natureza combinada dermatoneurológicas ${ }^{5-7}$.

Nas últimas décadas, sobretudo a partir da implantação da poliquimioterapia, a carga da hanseníase vem declinando consideravelmente, o que resultou na eliminação da doença como problema de saúde pública global no ano 2000, restando determinados países com elevada carga e que não atingiram a eliminação em nível nacional, dentre os quais, o Brasil. Mesmo com todos os esforços, somente em 2014, 213.899 casos novos foram registrados no mundo, sendo $94 \%$ desses pacientes oriundos de treze nações ${ }^{8,9}$.

No cenário global, o Brasil ocupa a segunda posição em número de casos novos e a primeira em coeficiente de prevalência, sendo o único que possui uma prevalência superior a 1/10 mil habitantes e que, portanto, ainda não atingiu a meta de eliminação da doença. Em 2016, essa prevalência foi de $1,1 / 100$ mil. Nesse mesmo ano, o país registrou 25.218 casos novos de hanseníase na população geral $(12,23$ casos novos/100 mil habitantes), sendo 1.696 (6,72\%) em menores de 15 anos (3,63/100 mil habitantes) $)^{9,10}$.

No Brasil, a distribuição espacial da hanseníase segue um padrão heterogêneo. Enquanto a doença tem baixa prevalência na região o Sul, em outras, como no Centro-oeste, Norte e Nordeste, a magnitude da endemia revela estar longe de ser vencida $^{10,11}$. Todas estas regiões possuem carac- terísticas socioeconômicas, históricas e culturais singulares ${ }^{12}$, o que nos faz pensar sobre a importância desses fatores na determinação social da hanseníase.

A Bahia registrou 2.077 casos novos de hanseníase no ano de 2016, expressando um coeficiente de detecção geral de 13,60/100 mil. Desse total, 116 indivíduos tinham idade inferior a 15 anos, manifestando um coeficiente de 3,16/100 mil. Quando comparado aos demais estados da região Nordeste, a Bahia ocupou a segunda posição em número absoluto de casos novos e a sexta quando analisados os coeficientes de detecção geral e em menores de 15 anos $^{10}$.

Muitos estudos, desde a década de 1930, têm posto em evidência a complexidade da relação entre a ocorrência da hanseníase e seus determinantes sociais ${ }^{11,13}$. A dinâmica dessa teia mediadora necessita ainda ser melhor compreendida, tendo em vista que não se trata de uma relação puramente linear e de causa e efeito. $\mathrm{O}$ olhar estatístico, epidemiológico e histórico-social apurado sobre o fenômeno de adoecimento pode ajudar a esclarecer essas múltiplas mediações e assim contribuir para que o Brasil possa alcançar a tão necessária eliminação da doença.

Desse modo, este trabalho teve como objetivo analisar a distribuição espacial da hanseníase no estado da Bahia relacionando-a com indicadores socioeconômicos.

\section{Materiais e métodos}

Trata-se de um estudo de agregado observacional (ecológico), com desenho de múltiplos grupos, adotando-se como unidade de análise o estado da Bahia e seus 417 municípios. A investigação envolveu a análise de três indicadores epidemiológicos de monitoramento da hanseníase na Bahia, registrados no período de 2001 a 2015: coeficientes de detecção de hanseníase na população geral, coeficiente de detecção em menores de 15 anos e a taxa de casos novos de hanseníase com grau II de incapacidade física no momento do diagnóstico. Os dados necessários para o cálculo desses indicadores foram obtidos do Sistema Nacional de Agravos de Notificação (SINAN) e a metodologia adotada obedeceu ao que preconiza a portaria $\mathrm{n}^{\circ}$ $149 / 2016^{14}$. Salienta-se que esses indicadores atuaram como variáveis dependentes.

As variáveis independentes foram selecionadas considerando uma ampla revisão da literatura especializada ${ }^{15-25}$, a fim de identificar os principais fatores associados à ocorrência da 
hanseníase, sendo escolhidas: densidade demográfica (DENSIDEMO), proporção da população urbana (\%POPURB), domicílio coletivo com morador (DOMICOLE), proporção de indivíduos com 60 anos ou mais na população (\%POP60+), proporção de indivíduos com 15 anos ou mais analfabetos (\%POP15+ANALF), proporção de domicílios com saneamento inadequado (\%DOMSINAD), valor médio mensal da renda per capita (RENDPERCAPT), proporção de extremamente pobres (\%EXTPOBRES), número de domicílios com densidade maior do que três pessoas por dormitório (DOM3PPDOR), nível de ocupação de indivíduos com 10 anos ou mais (NIVOCUP10+), domicílios sem rendimento (DOMSEMREND), família composta por seis ou mais conviventes no domicílio (FA6+PDPP), pessoa responsável e cônjuge sem rendimento (PRESCSEMREND), proporção de unidades domésticas unipessoais (\%UNIPESSOAIS), número de domicílios particulares permanentes ligados à rede geral de abastecimento de água (DPPABAST), número de domicílios particulares permanentes sem banheiro de uso exclusivo do domicílio (DPPSEMBAN) e número de domicílios particulares permanentes com lixo coletado (DPPLIXOCOL). Os indicadores selecionados utilizados foram obtidos do Censo Demográfico de $2010^{26}$.

A primeira fase da análise consistiu na suavização dos indicadores epidemiológicos (variáveis dependentes) pelo Modelo Bayesiano Empírico Local. A suavização justificou-se pela existência de um grande número de municípios pequenos e com dados que sugerem subnotificação $0^{25,27-33}$. Em seguida, foi conduzida uma análise da dependência espacial utilizando a estatística de Moran Global e Local. Os municípios foram classificados em: Q1- Alto/alto (valores positivos e médias positivas- alta prioridade), Q2- Baixo/baixo (valores negativos e médias negativas- baixa prioridade), Q3-alto/baixo (valores positivos e médias negativas- prioridade intermediária) e Q4-baixo/ alto (valores negativos e médias positivas- prioridade intermediária $)^{34,35}$.

$\mathrm{Na}$ etapa seguinte, foi utilizada regressão linear multivariada (método Backward) ${ }^{36}$. Para garantir a normalidade dos dados, adotou-se a conversão em escala log. A análise de Moran dos resíduos do modelo OLS (Ordinary Least Squares) foi realizada para a identificação da necessidade de incorporação de componente espacial. Uma vez constatada a dependência, os testes multiplicadores de Lagrange foram utilizados para a escolha do melhor modelo (Spatial Error
Model ou Spatial Lag Model) em consonância com o esquema decisório proposto por Luc Anselin. No Spatial Error Model, os efeitos espaciais são ruídos que devem ser removidos. Já o Spatial Lag Model atribui à variável resposta $\mathrm{Y}$ a autocorrelação espacial ignorada ${ }^{37}$. Os resíduos foram submetidos ainda à avaliação da normalidade (teste de Jarque-Bera) e homocedasticidade (teste de Breusch-Pagan).

Ao final, adotou-se os seguintes mecanismos para a avaliação da qualidade do modelo: Akaike (AIC), bayesiano de Schwarz (BIC), $\mathrm{R}^{2}$, log de Verossimilhança e estatística I Moran dos resíduos. Considerou-se como melhor modelo aquele cujos valores de AIC e BIC foram menores, o Log de Verossimilhança e o $\mathrm{R}^{2}$ foram maiores e os resíduos apresentaram independência espacial ${ }^{38}$.

As análises foram realizadas utilizando o software Terra View 4.2.2, QGis 2.14.11 e GeoDa 1.18.10. As malhas territoriais foram obtidas no site do IBGE.

O estudo foi aprovado pelo Comitê de Ética em Pesquisa da Universidade Federal de Alagoas e executado entre agosto de 2017 e janeiro de 2018.

\section{Resultados}

Entre 2001 e 2015, com base nos dados do SINAN, foram notificados 42.227 casos novos de hanseníase no estado da Bahia, sendo a média de $2.815,1$ casos/ano. Desse total, $8,1 \%(\mathrm{n}=3.430)$ dos doentes eram menores de 15 anos, sendo a média de 228,7 casos/ano. O grau II de incapacidade física no momento do diagnóstico esteve presente em 4,8\% ( $\mathrm{n}=2.032)$ dos indivíduos diagnosticados.

O coeficiente de detecção geral médio do período estudado foi de 19,98/100 mil (endemicidade alta - entre 10 e 19,99/100 mil), com pequena variação entre o início e o final da série temporal (16,71/100 mil em 2001 e 16,31/100 mil em 2015). Já em menores de 15 anos, o coeficiente de detecção foi de 5,66/100 mil (endemicidade muito alta - entre 5 e 9,99/100 mil). Enquanto em 2001 foram registrados 4,71 casos novos em menores de 15 anos por 100 mil habitantes, em 2015 esse coeficiente alcançou 5,88/100 mil. A taxa de casos com grau II de incapacidade física média foi de $0,95 / 100$ mil, sendo $0,67 / 100$ mil em 2001 e 0,83/100 mil em 2015.

A modelagem espacial mostrou um padrão de heterogeneidade na distribuição espacial da hanseníase na Bahia, constatada pelo índice de 
Moran. Na análise comparativa dos indicadores brutos com os suavizados pelo modelo bayesiano empírico local, o número de municípios classificados como hiperendêmicos (mais de 40 casos por 100 mil habitantes) passou de 29 (6,9\%) para 39 (9,3\%). Paralelamente, observou-se redução do número de municípios classificados como de endemicidade baixa (menos de 2 casos por 100 mil habitantes). O Moran Map desse indicador mostrou que o número de municípios situados no quadrante Q1 (considerados prioritários) passou de $35(8,4 \%)$ para 42 (10,7\%). No mapa, observou-se que esses municípios estão concentrados no eixo norte-oeste e na região sul do estado (Figura 1).

Já no que diz respeito à comparação entre o coeficiente de detecção em menores de 15 anos bruto e o suavizado, também se observou um aumento do número de municípios classificados como de endemicidade alta (2,5 a 4,99 casos por 100 mil habitantes), passando de 54 (12,9\%) para 73 (17,5\%). No Moran Map, o número de municípios situados no quadrante Q1 quase dobrou de valor, passando de $23(5,5 \%)$ para $45(10,8 \%)$. Do mesmo modo que o indicador anterior, observou-se que esses municípios concentraram-se no eixo norte-oeste e na região sul do estado (Figura 1).

Quanto à taxa de casos novos com grau II de incapacidade física no momento do diagnóstico, foi constatado um comportamento parcialmente diferente, caracterizado pelo deslocamento do número de municípios com algum grau de endemicidade para o número de municípios sem nenhum caso. Isto decorre do grande número de municípios sem notificação do agravo circundando outros onde ocorreu a notificação. Por outro lado, no Moran Map houve incremento do número de municípios situados no quadrante Q1, passando de $20(4,8 \%)$ para $31(7,4 \%)$, estando situados nas regiões norte, nordeste e sul (Figura 1).

No modelo de regressão tendo como variável dependente o coeficiente de detecção na população geral, sete indicadores foram associados inicialmente: DENSIDEMO, \%POPURB, RENDAPERCAPT, \%EXTRPOBRES, DOM3PPDOR, NIVOCUP10+, \%UNIPESSOAIS. Nota-se que as variáveis que permaneceram no modelo foram aquelas relacionadas à urbanização e à aglomeração populacional (\%POPURB; DOM3PPDOR; DENSIDEMO) e às condições socioeconômicas (\%EXTRPOBRES; NIVOCUP10+; RENDPERCAPIT). Além disso, municípios com maior proporção de moradias unipessoais tiveram associa- ção inversa com o coeficiente de detecção geral (\%UNIPESSOAIS). Para o coeficiente de deteç̧ão em menores de 15 anos, três variáveis apresentaram significância: \%POP60+, RENDPERCAPIT e NIVOCUP10+. Nota-se que populações mais envelhecidas estavam inversamente relacionadas ao desfecho (\%POP60+), assim como o nível de ocupação mais elevado (NIVOCUP10+). Para o indicador taxa de casos novos com grau II de incapacidade física, apenas a variável \%POP15+ANALF apresentou significância. Nota-se neste modelo uma associação entre o analfabetismo e a ocorrência do agravo (Tabela 1).

No modelo gerado para o coeficiente de detecção em menores de 15 anos, o teste de normalidade de Jarque-Bera dos resíduos mostrou distribuição diferente da normal $(p<0,001)$ e o teste de Breusch-Pagan mostrou heterocedasticidade $(p<0,001)$. Em razão desses problemas, esse indicador foi excluído das análises seguintes. Os modelos gerados para os demais indicadores apresentaram resíduos com distribuição normal e homocedasticidade (Coeficiente de detecção de hanseníase na população geral: Jarque-Bera, $p=$ 0,562; Breusch-Pagan, $p=0,500 /$ Taxa de casos novos com grau II de incapacidade física: Jarque -Bera, $p=0,363$; Breusch-Pagan, $p=0,198$ ).

A dependência espacial dos resíduos dos modelos para os dois indicadores foi confirmada pela estatística I de Moran. Obteve-se I igual a $0,422(p<0,001)$ para o coeficiente de deteç̧ão geral e I igual a 0,309 $(p<0,001)$ para a taxa de casos novos com grau II de incapacidade física. Os testes multiplicadores de Lagrange, versão robusta, mostraram que o melhor modelo espacial a ser aplicado era o Spatial Lag Model para ambos os indicadores $(p<0,001$ para o coeficiente de detecção de hanseníase na população geral e $p=$ 0,004 para a taxa de casos novos com grau II de incapacidade física).

Ao aplicar o Spatial Lag Model para o coeficiente de detecção de casos novos da população geral, as variáveis NIVOCUP10+ e \%UNIPESSOAIS não apresentaram significância estatística e foram excluídas do modelo final, mantendo-se cinco variáveis. Quanto ao indicador taxa de casos novos com grau II de incapacidade física, a variável \%POP15+ANALF manteve-se no modelo. A Tabela 2 mostra os resultados do Spatial Lag Model para os dois indicadores analisados.

$\mathrm{Na}$ avaliação dos modelos, conforme expresso na Tabela 3, constatou-se que o Spatial Lag melhorou a qualidade dos resultados, uma vez que houve um aumento importante do coeficiente de determinação $\left(\mathrm{R}^{2}\right)$, acompanhado de elevação 

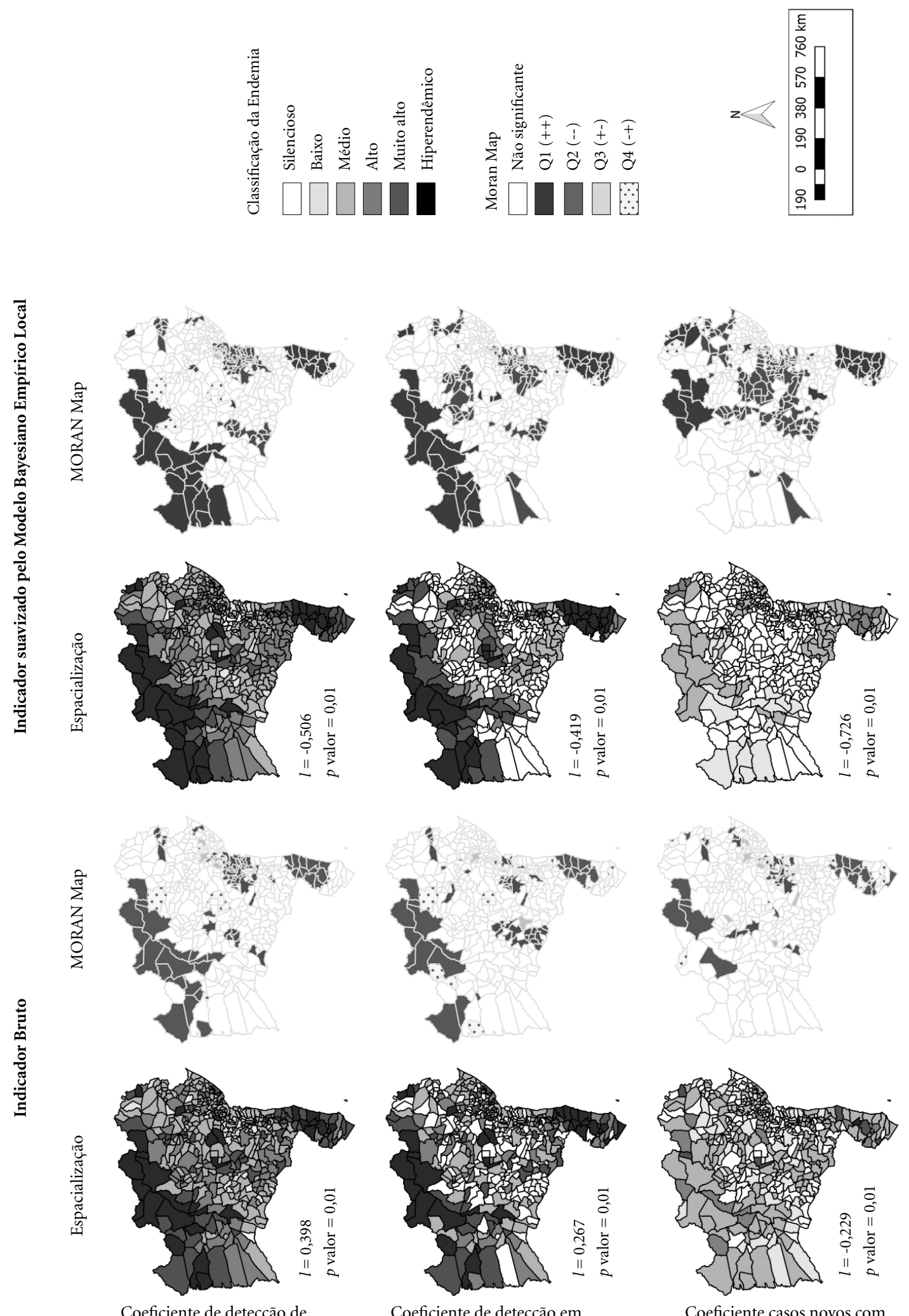
Hanseníase na população geral

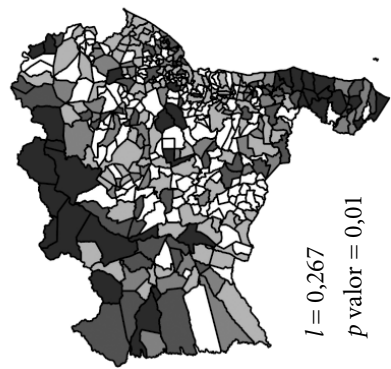

Coeficiente de detecção em menores de 15 anos (por 100 mil habitantes)

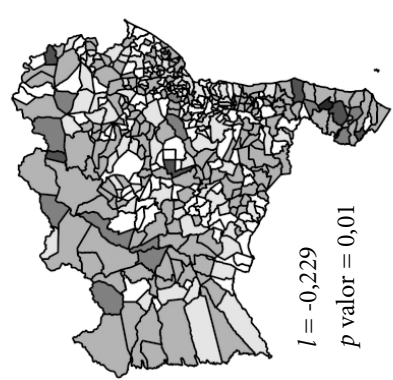

Coeficiente casos novos com grau II de incapacidade física (por 100 mil habitantes)

Figura 1. Indicadores epidemiológicos de magnitude da hanseníase brutos, suavizados pelo Modelo Bayesiano Empírico Local e Moran Map. Bahia-Brasil, 2001-2015. 
do Log de Verossimilhança e redução nos valores dos critérios de Akaike e Schwarz. Adicionalmente, de acordo com a Figura 2, a incorporação da

Tabela 1. Resultados do modelo de regressão multivariada (OLS) para $\log$ dos indicadores epidemiológicos suavizados: coeficiente de detecção de casos novos de hanseníase na população geral, coeficiente de detecção em menores de 15 anos e taxa de casos novos com grau II de incapacidade física.

\begin{tabular}{|c|c|c|}
\hline Variável & Coeficiente & p valor \\
\hline \multicolumn{3}{|c|}{$\begin{array}{l}\text { Coeficiente de detecção de } \\
\text { hanseníase na população } \\
\text { geral }\end{array}$} \\
\hline Constante & 0,964804 & $<0,001$ \\
\hline DENSIDEMO $^{a}$ & $-0,000697$ & $<0,001$ \\
\hline$\% P^{2}$ PURB ${ }^{\mathrm{b}}$ & 0,003452 & 0,021 \\
\hline RENDAPERCAPIT $^{c}$ & 0,001932 & $<0,001$ \\
\hline \%EXTRPOBRES ${ }^{d}$ & 0,006872 & 0,004 \\
\hline DOM3PPDOR $^{e}$ & 0,000004 & 0,006 \\
\hline NIVOCUP10 $+^{\mathrm{f}}$ & $-0,013267$ & $<0,001$ \\
\hline \%UNIPESSOAIS & $-0,023791$ & 0,005 \\
\hline \multicolumn{3}{|c|}{$\begin{array}{l}\text { Coeficiente de detecção em } \\
\text { menores de } 15 \text { anos }\end{array}$} \\
\hline Constante & 0,878027 & $<0,001$ \\
\hline$\% \mathrm{POP} 60+^{\mathrm{h}}$ & $-0,039240$ & $<0,001$ \\
\hline RENDPERCAPIT $^{c}$ & 0,000340 & $<0,001$ \\
\hline NIVOCUP10+ ${ }^{\mathrm{f}}$ & $-0,011842$ & $<0,001$ \\
\hline \multicolumn{3}{|c|}{$\begin{array}{l}\text { Taxa de casos novos com } \\
\text { grau II de incapacidade física }\end{array}$} \\
\hline Constante & $-0,129782$ & 0,008 \\
\hline$\%$ POP15+ANALF ${ }^{\mathrm{i}}$ & 0,004955 & $<0,001$ \\
\hline \multicolumn{3}{|c|}{ 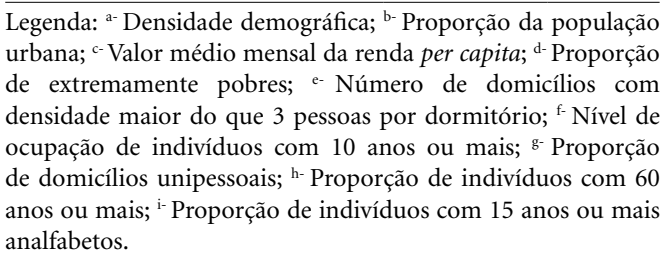 } \\
\hline
\end{tabular}

regressão espacial com efeitos globais eliminou a dependência espacial dos resíduos nos dois modelos (I Moran $=-0,046$ para o modelo do coeficiente de detecção de hanseníase na população geral e I Moran $=-0,050$ para o da taxa de casos novos com grau II de incapacidade).

\section{Discussão}

O estudo da distribuição espacial da hanseníase possibilitou aprofundar o conhecimento sobre a cadeia epidemiológica da doença no estado da

Tabela 2. Resultados do Spatial Lag Model para log dos indicadores epidemiológicos coeficiente de detecção de hanseníase na população geral e taxa de casos novos com grau II de incapacidade física suavizados pelo modelo Bayesiano Empírico Local.

\begin{tabular}{|c|c|c|}
\hline Variável & Coeficiente & p valor \\
\hline \multicolumn{3}{|c|}{$\begin{array}{l}\text { Coeficiente de detecção de } \\
\text { hanseníase na população } \\
\text { geral }\end{array}$} \\
\hline Constante & -0.503932 & $<0,001$ \\
\hline DENSIDEMO $^{\mathrm{a}}$ & -0.000412 & 0,001 \\
\hline$\%$ POPURB ${ }^{\mathrm{b}}$ & 0.003156 & 0,001 \\
\hline RENDAPERCAPIT $^{c}$ & 0.001085 & 0,001 \\
\hline$\%$ EXTRPOBRES $^{\mathrm{d}}$ & 0.006112 & $<0,001$ \\
\hline DOM3PPDOR $^{e}$ & 0.000003 & 0,017 \\
\hline \multicolumn{3}{|c|}{$\begin{array}{l}\text { Taxa de casos novos com } \\
\text { grau II de incapacidade física }\end{array}$} \\
\hline Constante & -0.064866 & 0.022 \\
\hline$\% \mathrm{P} 15+$ ANALF $^{\mathrm{f}}$ & 0.002580 & 0.024 \\
\hline
\end{tabular}

Legenda: ${ }^{\text {a- }}$ Densidade demográfica; ${ }^{\text {b- }}$ Proporção da população urbana; ${ }^{\mathrm{c}-}$ Valor médio mensal da renda per capita; ${ }^{\mathrm{d}-}$ Proporção de extremamente pobres; ${ }^{-}$- Número de domicílios com densidade maior do que 3 pessoas por dormitório; ${ }^{\mathrm{f}}$ Proporção de indivíduos com 15 anos ou mais analfabetos.

Tabela 3. Comparação dos modelos de regressão clássica (OLS) e Spatial lag com base no coeficiente de determinação $\left(\mathrm{R}^{2}\right)$, Log de Verossimilhança, critérios de Akaike (AIC) e Schwarz (SBC) para log dos coeficientes de detecção de hanseníase na população geral e taxa de casos novos com grau II de incapacidade física suavizados pelo modelo Bayesiano Empírico Local.

\begin{tabular}{lcccc}
\hline \multicolumn{1}{c}{ Critério } & \multicolumn{2}{c}{$\begin{array}{c}\text { Coeficiente de detecção de } \\
\text { hanseníase na população geral }\end{array}$} & $\begin{array}{c}\text { Taxa de casos novos com grau II } \\
\text { de incapacidade física }\end{array}$ \\
\hline & OLS & $\begin{array}{c}\text { Spatial Lag } \\
\text { Model }\end{array}$ & OLS & $\begin{array}{c}\text { Spatial Lag } \\
\text { Model }\end{array}$ \\
\hline $\mathrm{R}^{2}$ & 0.137783 & 0.535054 & 0.028310 & 0.285517 \\
Log de Verossimilhança & -247.452 & -135.979 & 163.527 & 216.988 \\
Akaike (AIC) & 510.904 & 285.958 & -321.055 & -427.977 \\
Schwarz (SBC) & 543.169 & 314.189 & -308.955 & -415.877 \\
\hline
\end{tabular}



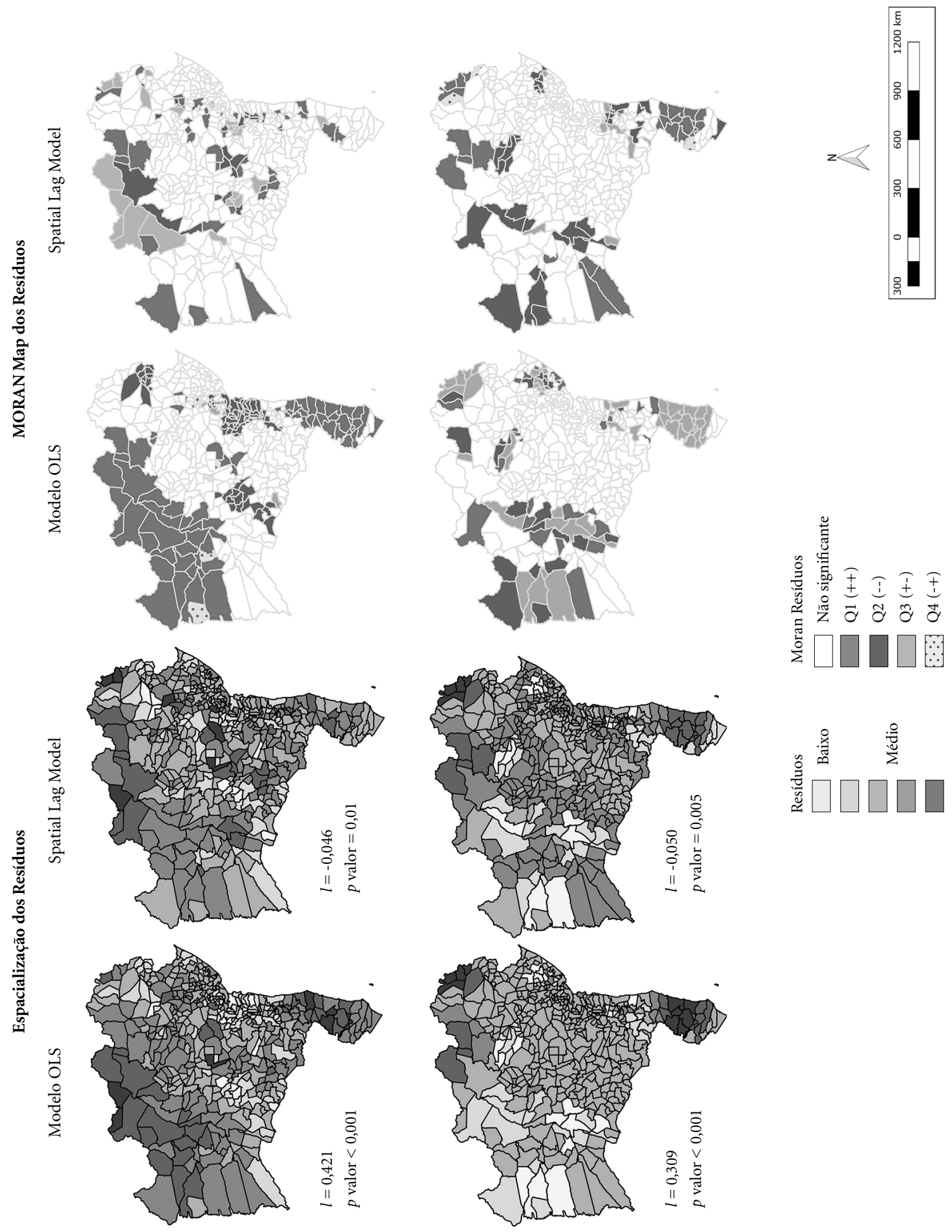

Coeficiente de detecção de Hanseníase na população geral

Coeficiente de casos novos com grau II de incapacidade física (por 100 mil habitantes)

Figura 2. Distribuição espacial e Moran Map dos resíduos dos modelos OLS e Spatial Lag para os coeficientes de detecção de hanseníase na população geral e taxa de casos novos com grau II de incapacidade física no diagnóstico. Bahia-Brasil, 20012015. 
Bahia. A primeira constatação é derivada da aplicação da modelagem Bayesiana e da estatística de Moran. Esse conjunto de métodos de análise espacial possibilitou mostrar que os indicadores epidemiológicos observados são menores do que o esperado, exceto para o grau II de incapacidade. Além disso, o número de municípios de alta prioridade foram maiores após a modelagem dos três indicadores.

Esse cenário dissonante entre o observado e o esperado tem como potencial explicação a existência de problemas operacionais persistentes nos municípios. Alguns dos problemas mais recorrentes que acabam por comprometer o acompanhamento do processo de eliminação da doença como problema de saúde pública ${ }^{39-42}$, são: subnotificação, falhas na digitação de dados, pouca cobertura das equipes de saúde, falta de profissionais capacitados para o diagnóstico e o acompanhamento dos doentes, deficiência dos setores de vigilância e pouco investimento em ações sustentáveis.

A segunda constatação refere-se à distribuição heterogênea da doença no espaço geográfico, isto é, ela não ocorre ao acaso. Esse padrão não aleatório corrobora para a validação da hipótese de que a hanseníase está associada a uma combinação de fatores geográficos, sociais, econômicos e culturais que expressam o modo de vida da população. Nesse sentido, a distribuição geográfica heterogênea já foi mostrada em estudos desde a escala mundial até a intramunicipal ${ }^{21,43-49}$.

A concentração dos maiores coeficientes de detecção de hanseníase na população geral e em menores de 15 anos no eixo norte-oeste e região sul do estado indica que a transmissão da doença está ativa nesses locais. Importante destacar que o adoecimento da população infantil é um dos mais sensíveis indicadores capazes de sinalizar a circulação do Micobacterium leprae numa região ${ }^{14,19,20}$. Esse cenário pode ser influenciado pelo padrão de ocupação desses territórios e por suas implicações nas condições materiais de vida de pessoas. O primeiro aspecto a ser destacado é o processo migratório, apresentado por diferentes estudos como importante elemento da cadeia de transmissão da hanseníase $e^{27,45,50,51}$.

A migração no estado da Bahia configurou-se como um processo histórico-social caracterizado tanto pela saída da população do campo para ocupar as cidades (êxodo rural), quanto pela migração de grandes contingentes populacionais vindos de todas as partes do país para regiões com potencial produtivo, com destaque para a fruticultura irrigada no norte do estado - na re- gião do Vale do São Francisco -, a produção de soja no Oeste e a produção cacaueira no Sul ${ }^{52-54}$.

No caso da região Sul do estado, diferentemente das demais áreas, um processo bastante peculiar se estabeleceu em razão das perdas produtivas nas plantações de cacau em decorrência de pragas, forçando um intenso êxodo rural para as cidades, principalmente para Ilhéus e Itabu$\mathrm{na}^{53}$. Como fruto do processo migratório houve o aumento da população urbana, que neste estudo esteve associada com a ocorrência da hanseníase. Resultados semelhantes foram observados em estudos realizados no Maranhão ${ }^{24}$, no Ceará ${ }^{55}$ e no Tocantins ${ }^{45}$. Paralelamente, o crescimento urbano desordenado também é apontado por Imbiriba et al. ${ }^{20}$ como um importante elemento envolvido na expansão da endemia na cidade de Manaus, no Amazonas.

A relação inversa entre a densidade demográfica e o coeficiente de detecção de hanseníase na população geral também foi evidenciada em estudo realizado no Rio de Janeiro por Gracie et al. ${ }^{27}$. Segundo os autores, esse achado justifica-se pelo fato de que municípios de porte populacional pequeno, situados em extensão territorial considerável, terem elevados coeficientes de detecção. No caso da Bahia, por exemplo, dos dez municípios com os maiores coeficientes de detecção geral (Remanso, Belmonte, Itabela, Guaratinga, Andaraí, Barreiras, Sobradinho, Santa Rita de Cássia, Campo Alegre de Lourdes e Eunápolis), oito possuíam, segundo o censo de 2010, uma população inferior a 45 mil habitantes.

Vivendo nas periferias das cidades, em condições inapropriadas de moradia, com pouco acesso aos serviços municipais e em alto grau de vulnerabilidade, o risco de contrair a doença eleva-se consideravelmente ${ }^{20}$. Sendo uma doença respiratória, cuja transmissão se dá de pessoa a pessoa, é justificável que quanto mais gente vivendo junto com um doente, maior o risco de transmissão. Nesse estudo, o número de domicílios com densidade maior do que três pessoas por dormitório mostrou associação positiva com ocorrência da hanseníase, sendo um componente importante do modelo. Por outro lado, quanto maior a proporção de domicílios unipessoais, menor foi a ocorrência da doença. Estudos de Freitas et al. ${ }^{21}$ e Souza et al. ${ }^{56}$ corroboram com esses achados, destacando que o risco de contaminação pelo $\mathrm{Mi}$ cobacterium leprae é elevado naqueles domicílios cuja média de moradores por cômodo e por domicílio particular permanente é maior.

Esse achado reforça a já conhecida importância do contato intradomiciliar na cadeia de 
transmissão da hanseníase ${ }^{57}$. Segundo Santos et al. ${ }^{58}$, o risco de um indivíduo sadio adquirir hanseníase tendo contato com um doente na família é 2,9 vezes maior do que um outro sem um enfermo no seio familiar. Importante destacar que a vigilância dos contatos intradomicilares, por si só, pouco tem contribuído para o enfretamento ao problema. Nesse sentido, é necessária uma expansão do conceito de contatos, uma vez que ele deve extrapolar a dimensão domiciliar e alcançar o meio social no qual os sujeitos estão inseridos. Dada a relevância dessa relação, o Ministério da Saúde tem enfatizado a importância do adequado exame de contatos domiciliares e sociais como estratégia para a interrupção da transmissão e a redução da carga da doença no país ${ }^{14}$.

Outro determinante do adoecimento que merece reflexão é a renda per capita. Municípios com maior renda apresentaram também os maiores coeficientes de detecção de casos novos. Provavelmente, porque estes municípios possuem uma melhor rede de serviços de saúde, que pode ampliar o acesso da população mais pobre a estes, tornando o diagnóstico mais fácil. Essa ampliação do acesso aumenta o número de casos diagnosticados e reduz a prevalência oculta, o que resulta em coeficientes maiores.

A análise da renda como determinante da hanseníase, dada a sua complexidade, merece uma análise pormenorizada. $\mathrm{O}$ aumento da renda per capita municipal, por si só, não significa que esta esteja distribuída uniformemente por toda a população. A associação encontrada entre a proporção de extremamente pobres e a ocorrência da hanseníase indicam haver desigualdade na distribuição de renda nos municípios com maior carga dessa enfermidade, corroborando com a literatura que destaca a pobreza como um dos mais importantes determinantes sociais ${ }^{21,59}$.

Muitos autores têm apontado a desigualdade de renda como um fator fortemente associado à hanseníase. Freitas et al. ${ }^{21}$, em estudo em todo o território brasileiro, mostraram que municípios com Índice de Gini maior apresentavam também maior risco de transmissão. Souza et al. ${ }^{59}$, em estudo conduzido no estado da Bahia, mostraram associação positiva entre a ocorrência da doença e a desigualdade de renda. Kerr-Pontes et al. ${ }^{18}$ evidenciaram que fatores que expressam pobreza ampliam o risco de transmissão: habitação com piso de barro, baixa frequência de mudança de roupas de cama, privação alimentar e baixo nível de escolaridade.
A ascensão econômica das famílias impacta direta ou indiretamente em outros determinantes sociais já comprovadamente envolvidos na manutenção da cadeia epidemiológica de transmissão da doença, como educação, saneamento, estado nutricional e condições de moradia $^{18,21,56,60,61}$. Neste estudo, a taxa de analfabetismo foi um determinante importante associado às taxas mais elevadas de casos novos de hanseníase com grau II de incapacidade física no momento do diagnóstico, sugerindo que este é tardio e que há prevalência oculta.

Importante salientar que a luta contra a pobreza ganhou espaço no cenário mundial com a agenda 2030 de desenvolvimento sustentável. Instituída em 2015, o plano definiu 17 objetivos e 169 metas a serem alcançadas até 2030, versando sobre diferentes dimensões dos determinantes sociais. Advogamos que o compromisso assumido acarretará em redução das doenças negligenciadas em todo o mundo ${ }^{62}$.

Todas as interpretações conduzidas neste trabalho levaram em consideração dois conjuntos de limitações. $\mathrm{O}$ primeiro diz respeito às próprias dos estudos ecológicos: dificuldade de inferência de resultados e de controlar os fatores de confundimento, impossibilidade de associar exposição e doença e o viés ecológico (falácia ecológica) ${ }^{63}$.

O segundo conjunto refere-se ao uso de dados secundários provenientes dos sistemas de informação em saúde. Muitas vezes, a qualidade da informação tem sido questionada em vários municípios, sobretudo naqueles cujas condições operacionais dos serviços de vigilância da hanseníase não são adequadas. Possivelmente, algumas contradições observadas no estudo possam ter decorrido da duvidosa qualidade dos dados disponíveis. A modelagem bayesiana e os modelos de regressão espacial contribuíram para construir um modelo final robusto capaz de identificar os principais fatores sociais e econômicos envolvidos na manutenção da cadeia de transmissão no estado da Bahia.

Ressalta-se que o conhecimento desses fatores pode contribuir para o desenvolvimento de planos e estratégias de eliminação que considerem a hanseníase como um problema complexo, dinâmico e multifatorial. Essa compreensão fortalece a ideia de que a identificação e o tratamento de doentes, por si só, não são capazes de acabar com a hanseníase. Outras frentes de trabalho devem a ser incorporadas a fim de intervir sobre os determinantes do adoecimento. 


\section{Colaboradores}

CDF Souza, MAFM Magalhães e CF Luna: participaram da concepção e o delineamento ou a análise e interpretação dos dados, redação do artigo e a sua revisão crítica e aprovação da versão a ser publicada. RA Medronho e FGB Santos: participaram da redação do artigo e a sua revisão crítica e aprovação da versão a ser publicada.

\section{Referências}

1. Macinko J, Starfield B. Annotated bibliography on equity in health, 1980-2001. Int J Equity Health 2002; 1(1):1.

2. Buss PM, Pelegrini Filho AA. Saúde e Seus determinantes sociais. Physis 2007; 17(1):77-93.

3. Carvalho AI, Buss PM. Determinantes sociais na Saúde, na doença e na intervenção. In: Giovanella L, Escorel S, Lobato LVC, Noronha JC, Carvalho AI. Politica e Sistema de Saúde no Brasil. $2^{\circ}$ ed. Rio de Janeiro: Fiocruz; 2012. p. 121-142.

4. Borde E, Hernández-Álvarez M, Porto MFS. Uma análise crítica da abordagem dos Determinantes Sociais da Saúde a partir da medicina social e saúde coletiva latino-americana. Saúde Debate 2015; 39(106):841-854.

5. Lockwood DN, Lucas SB, Desikan KV, Ebenezer G, Suneetha S, Nicholls P. The histological diagnosis of leprosy type 1 reactions: identification of key variables and an analysis of the process of histological diagnosis. J Clin Pathol 2008; 61(5):595-600.

6. Pires CAA, Miranda MFR, Bittencourt MJS, Brito AC, Xavier MB. Comparison between histopathologic features of leprosy in reaction lesions in HIV coinfected and non-coinfected patients. An Bras Dermatol 2015; 90(1):27-34.

7. Bahia El Idrissi N, Das PK, Fluiter K, Rosa PS, Vreijling J, Troost D, Morgan BP, Baas F, Ramaglia V. M. leprae components induce nerve damage by complemente activation: identification of lipoarabinomannanas the dominant complement activator. Acta Neuropathol 2015; 129(5):653-667.

8. White C, Franco-Paredes C. Leprosy in the 21st century. Clin Microbiol Rev 2015; 28(1):80-94.

9. World Health Organization (WHO). Global Leprosy Strategy: Accelerating towards a leprosy-free world. Geneva: WHO; 2016.

10. Brasil. Ministério da Saúde (MS). Registro ativo: número e percentual, casos novos de hanseníase: número, coeficiente e percentual, faixa etária, classificação operacional, sexo, grau de incapacidade, contatos examinados, por estado e regiões, Brasil, 2016. Brasília: MS. [acessado 2017 Mar 25]. Disponível em: http:// portalsaude.saude.gov.br/index.php/o-ministerio/ principal/leia-mais-o-ministerio/705-secretaria-svs/ vigilancia-de-a-a-z/hanseniase/11298-situacao-epidemiologica-dados.

11. Silva CLM, Fonseca SC, Kawa H, Palmer DOQ. Spatial distribution of leprosy in Brazil: a literature review. Rev. Soc. Bras. Med. Trop 2017; 50(4):439-449.

12. Barros RP, Mendonça SRP, Duarte RPN. Bem-Estar, Pobreza e Desigualdade de Renda: Uma avaliação da evolução histórica e das disparidades regionais. Brasília: Instituto de Pesquisa Econômica Aplicada; 1998.

13. Mencaroni DA, Pinto Neto JM, Villa TCS, Oliveira MHP. Análise espacial da endemia hansênica na área urbana do município de Fernandópolis/SP. Hansen. int 2004; 29(1):12-20.

14. Brasil. Ministério da Saúde (MS). Diretrizes para vigilância, atenção e eliminação da Hanseníase como problema de saúde pública: manual técnico-operacional. Brasília: MS; 2016. 
15. Andrade VLG, Sabroza PC, Araújo AJG. Fatores associados ao domicílio e à família na determinação da Hanseníase, Rio de Janeiro, Brasil. Cad Saude Publica 1994; 10(2):281-292.

16. Lapa T, Ximenes R, Silva NN, Souza W, Albuquerque MFM, Compozana G. Vigilância da hanseníase em Olinda, Brasil, utilizando técnicas de análise espacial. Cad Saude Publica 2001; 17(5):1153-1162.

17. Mencaroni DA. Análise espacial da endemia hansênica no município de Fernandópolis/SP [tese]. Ribeirão Preto: USP; 2003.

18. Kerr-Pontes LRS, Barreto ML, Evangelista CMN, Rodrigues LC, Heukelbach J, Feldmeier H. Socioeconomic, environment, and behavioural risk factors for leprosy in North-east Brazil: results of a case-control study. Int J Epidemiol 2006; 35(4):994-1000.

19. Santos AS, Castro DS, Falqueto A. Fatores de risco para transmissão da Hanseníase. Rev Bras Enferm 2008; 61(n. esp):738-743.

20. Imbiriba ENB, Silva Neto NA, Souza, WV, Pedrosa V, Cunha MG, Garnelo L. Desigualdade social, crescimento urbano e hanseníase em Manaus: abordagem espacial. Rev Saude Publica 2009; 43(4):656-665.

21. Freitas LRS, Duarte EC, Garcia LP. Leprosy in Brazil and its association with characteristics of municipalities: ecological study, 2009-2011. Trop Med Int Health 2014; 19(10):1216-1225

22. Cabral-Miranda W, Chiaravalloti Neto F, Barrozo LV. Socioeconomic and environmental effects in fluencing the development of leprose in Bahia, nort-east Brazil. Trop Med Int Health 2014; 19(12):1504-1514.

23. Duarte-Cunha M, Cunha GM, Souza-Santos R. Geographical heterogeneity in the analysis of factors associated with leprosy in an endemic area of Brazil: are we eliminating the disease? BMC Infec Dis 2015; 15:196.

24. Rangel MES. Dinâmica espacial e contingências socioambientais da hanseníase no estado do Maranhão: avaliação de riscos e vulnerabilidades em área hiperendêmica [tese]. São Paulo: Universidade de São Paulo; 2016.

25. Monteiro LD, Mota RMS, Martins-Melo FR, Alencar $\mathrm{CH}$, Heukelbach J. Determinantes sociais da hanseníase em um estado hiperendêmico da região Norte do Brasil. Rev Saude Publica 2017; 51(70):1-11.

26. Instituto Brasileiro de Geografia e Estatística (IBGE). Censo demográfico 2010. Características da população e dos domicílios: resultado do universo. Rio de Janeiro: IBGE; 2010.

27. Gracie R, Peixoto JNB, Soares FBR, Hacker MAVB. Análise da distribuição geográfica dos casos de hanseníase- Rio de Janeiro, 2001 a 2012. Cien Saude Colet 2017; 22(5):1695-1704.

28. Assunção RM, Barreto SM, Guerra HL, Sakurai E. Mapas de taxas epidemiológicas: uma abordagem Bayesiana. Cad Saude Publica 1998; 14(4):713-723.

29. Souza WV, Barcellos CC, Brito AM, Carvalho MS, Cruz OG, Albuquerque MFM, Alves KR, Lapa TM. Aplicação de modelo bayesiano empírico na análise espacial da ocorrência de hanseníase. Rev Saude Publica 2001; 35(5):474-480.
30. Catelan D, Lagazio C, Biggeri A. A Hierarchical Bayesian approach to multiple testing in disease mapping. Biome J 2010; 52(6):784-797.

31. Carvalho AXY, Silva GDM, Albuquerque PHM. Taxas bayesianas para o mapeamento de homicídios nos municípios brasileiros. Cad Saude Publica 2012; 28(7):1249-1262.

32. Nunes FG. Abordagem bayesiana na epidemiologia espacial de notificações de casos de dengue no Estado de Goiás. In: Anais do XVI Simpósio Brasileiro de Sensoriamento Remoto (SBSR) 2013; Foz do Iguaçu. p. 8554-8560.

33. Magalhães MAFM, Medronho RA. Análise espacial da Tuberculose no Rio de Janeiro no período de 2005 a 2008 e fatores socioeconômicos associados utilizando microdado e modelos de regressão espaciais globais. Cien Saude Colet 2017; 22(3):831-840.

34. Druck S, Carvalho MS, Câmara G, Monteiro AVM, organizadores. Análise espacial de dados geográficos. Brasília: Embrapa; 2004.

35. Santos SM, Souza WV. Introdução à Estatística Espacial para a Saúde Pública. Rio de Janeiro, Brasília: Fiocruz, Ministério da Saúde (MS); 2007. (Série B. Textos Básicos de Saúde-Capacitação e Atualização em Geoprocessamento em Saúde, v. 3).

36. Charnet R, Bonvino H, Freire CAL, Charnet EMR Análise de modelos de regressão linear com aplicações. Campinas: Unicamp; 1999.

37. Anselin L, Florax RJ. Small sample properties of tests for spatial dependence in regression models: Some further results. In: Anselin L, Florax RJ, editors. New Directions in Spatial Econometrics. Berlin: SpringerVerlag; 1995. p. 21-74.

38. Anselin L. Exploring Spatial Data with GeoDaTM: A Workbook. Santa Barbara: Centre for Spatially Integrated Social Science; 2005.

39. Cunha MD. Estatística espacial na investigação epidemiológica de fatores associados à detecção de casos de hanseníase no Rio de Janeiro [tese]. Rio de Janeiro: Fiocruz; 2012.

40. Paschoal VDA, Nardi SMT, Cury MRCO, Lombardi C, Virmond MCL, Silva RMDN, Paschoal JAA, Magalhães LC, Conte ECM, Kubota RMM, Soubhia RMC. Criação de banco de dados para sustentação da pós-eliminação em hanseníase. Cien Saude Colet 2011; 16(Supl. 1):1201-1210.

41. Salgado CG, Barreto JG, Silva MB, Frade MA, Spencer JS. What do we actually know about leprosy worldwide? Lancet Infect Dis 2016; 16(7):778.

42. Sousa GS, Silva RLF, Xavier MB. Hanseníase e Atenção Primária à Saúde: uma avaliação de estrutura do programa. Saúde debate 2017; 41(112):230-242.

43. Penna MLF. Tendência da taxa de detecção de hanseníase por 100.000 habitantes, nas unidades federadas do Brasil, 1980-2006 [documento técnico]. Brasília: Ministério da saúde; 2008.

44. Cury MRCO, Paschoal VDAP, Nardi SMT, Chierotti AP, Rodrigues Junior AL, Chiaravalloti-Neto F. Análise espacial da incidência de Hanseníase e fatores socioeconômicos associados. Rev Saude Publica 2012; 46(1):110-118 
45. Monteiro LD, Martins-Melo FR, Britol AL, Alencar $\mathrm{CH}$, Heukelbachl AJ. Padrões espaciais da hanseníase em um estado hiperendêmico no Norte do Brasil, 2001-2012. Rev Saude Publica 2015; 49(84):1-8.

46. Souza CDF, Rodrigues M. Magnitude, tendência e espacialização da hanseníase em menores de 15 anos no estado da Bahia, com enfoque em áreas de risco: um estudo ecológico. Hygeia 2015; 11(20):201-212.

47. Ramos ACV, Yamamura M, Arroyo LH, Popolin PM, Chiaravalloti Neto F, Palha PF, Uchoa SAC, Pieri FM, Pinto IC, Fiorati RC, Queiroz AAR, Belchior AS, Santos DT, Garcia MCC, Crispim JA, Alves LS, Berra TZ, Arcêncio RA. Spatial clustering and local risk of leprosy in São Paulo, Brazil. PLoS Negl Trop Dis 2017; 11(2):e0005381.

48. Chaves EC, Costa SV, Flores RLR, Neves EOS. Índice de carência social e hanseníase no estado do Pará em 2013: análise espacial. Epidemiol. Serv. Saúde 2017; 26(4):807-816.

49. Freitas LRS, Duarte EC, Garcia LP. Análise da situação epidemiológica da hanseníase em uma área endêmica no Brasil: distribuição espacial dos períodos 2001-2003 e 2010 - 2012. Rev Bras Epidemiol 2017; 20(04):702-713.

50. Magalhães MCC, Santos ES, Queiroz ML, Lima ML, Borges RCM, Souza MS, Ramos AN. Migração e Hanseníase no Mato Grosso. Rev Bras Epidemiol 2011; 14(3):386-397.

51. Murto C, Ariza L, Alencar CH, Chichava OA, Oliveira AR, Kaplan C, Silva LFM, Heukelbach J. Migration among individuals with leprosy: a population-based study in Central Brazil. Cad Saude Publica 2014; 30(3):487-501.

52. Costa DH, Mondardo ML. A modernização da agricultura no Oeste Baiano: Migração Sulista e novas territorialidades. Revista Geonorte 2013; 7(1):1347-1361.

53. Andrade MLN. Um estudo cultural do cacau com perspectivas para o turismo. [dissertação]. Ilhéus: Universidade Estadual de Santa Cruz; 2004.

54. Vilarim MA. A região de Petrolina - PE e Juazeiro BA: notas sobre as transformações locais, os sujeitos do campo e a migração. Anais do XI Encontro Nacional da Anpege. 2015. Presidente Prudente.2015.

55. Kerr-Pontes LR, Montenegro AC, Barreto ML, Werneck GL, Feldmeier H. Inequality and leprosy in Northeast Brazil: an ecological study. Int J Epidemiol 2004; 33(2):262-269.
56. Souza CDF, Franca Rocha WJSF, Lima RS. Distribuição espacial da endemia hansênica em menores de 15 anos em Juazeiro-Bahia, entre 2003 e 2012. Hygeia 2014; 10 (19):35-49.

57. Santos D. Avaliação do risco de adoecimento em contatos de pacientes de hanseníase considerando fatores individuais, domiciliares e contextuais [dissertação]. Rio de Janeiro: Fiocruz; 2012.

58. Santos AS, Castro DS, Falqueto A. Fatores de risco para transmissão da Hanseníase. Rev Bras Enferm 2008; 61 (esp):738-743.

59. Souza CDF, Luna CF, Magalhães MAFM. Modelagem espacial da hanseníase no estado da Bahia e seus determinantes sociais: Um estudo das iniquidades em saúde. An Bras Dermatol 2019; 94(2):182-191.

60. Feenstra SG, Nahar Q, Pahan D, Oskam L, Richardus JH () Recent Food Shortage Is Associated with Leprosy Disease in Bangladesh: A Case-Control Study. PLoS Negl Trop Dis 2011; 5(5):e1029.

61. Krawinkel MB. Interaction of Nutrition and Infections Globally: An Overview. Ann Nutr Metab 2012; 61(Supl. 1):39-45.

62. World Health Organization (WHO). Transforming our world: the 2030 Agenda for Sustainable Development. Geneva: WHO; 2015.

63. Medronho RA, Bloch KV, Luiz RR, Werneck GL. Epidemiologia. 2a ed. São Paulo: Atheneu; 2009.
Artigo apresentado em 09/03/2018

Aprovado em 26/11/2018

Versão final apresentada em 28/11/2018 